01,03,04,10

\title{
Коэффициенты усиления потока фононов в кристаллах с различным типом анизотропии упругой энергии
}

\author{
() И.Г. Кулеев, С.М. Бахарев \\ Институт физики металлов им. М.Н. Михеева, \\ Екатеринбург, Россия \\ E-mail: kuleev@imp.uran.ru
}

(Поступила в Редакцию 10 января 2018 г.

В окончательной редакции 25 января 2018 г.)

\begin{abstract}
Рассмотрено распространение фононных импульсов в кубических кристаллах с различным типом анизотропии упругой энергии. Получено замкнутое аналитическое выражение для коэффициента усиления потока фононов. Проанализированы особенности его зависимостей от типов кривизны изоэнергетических поверхностей для всех акустических мод, а также величины и знака параметров анизотропии.

Работа выполнена в рамках государственного задания по теме „Спин“ АААА-А18-118020290104-2 и проекту № 32-1.1.3.5 Программы фундаментальных исследований Президиума РАН согласно контракту Минобрнауки № 14.Z50.31.0025).
\end{abstract}

DOI: 10.21883/FTT.2018.07.46107.004

\section{1. Введение}

При изучении распространения фононных импульсов в упруго анизотропных кристаллах была отмечена резкая анизотропия пространственного распределения потока энергии акустических колебаний различных поляризаций (см. [1-6]). Исследования, проведенные в [1], показали, что амплитуды фононных импульсов в кристаллах $\mathrm{LiF}$ и $\mathrm{KCl}$ сильно зависят от их поляризаций и направления распространения. Для кристалла $\mathrm{LiF}$ в направлении [100] интенсивность потока поперечных фононов оказалась в 100 раз больше чем для продольных фононов, тогда как в кристаллах $\mathrm{KCl}$ - наоборот: амплитуда импульса поперечных фононов оказалась в 7 раз меньше, чем для продольных фононов. Для количественного описания этих эффектов Марис в работе [2] воспользовался моделью изотропной среды, как системой сравнения, и ввел понятие коэффициента усиления потока фононов $A^{\lambda}-$,enhancement factor", который также известен $[3,7,8]$, как ,amplification factor". Согласно [2] коэффициент $A^{\lambda}$ определяется как отношение потока фононов данной поляризации $\lambda$ для выбранного направления волнового вектора к соответствующему потоку в изотропной среде. При первом знакомстве с работами [2-7,9] возникает впечатление, что „фактор усиления“ имеет смысл коэффициента усиления плотности состояний фононов для упруго анизотропного кристалла относительно изотропной среды.

Однако, наличие конечных разрывов в коэффициентах усиления для поперечных мод в симметричных направлениях и обращение их в бесконечность в точках нулевой кривизны на изоэнергетической поверхности затрудняют использование этого метода для интерпретации экспериментальных данных по распространению фононных импульсов в упругоанизотропных кристаллах.
Для устранения неоднозначности коэффициента усиления медленных оперечных мод в направлении [100] в работе [2] было проведено усреднение фононного потока по детектору в виде круга, а в работе [7] — по прямоугольному детектору для всех симметричных направлений. На примере кристалла GaAs было показано, что в зависимости от размера и формы детектора коэффициенты усиления поперечных фононов могут изменяться в несколько раз. Причем, для вырожденных направлений [100] и [111] даже для бесконечно малых размеров детектора результат зависит от его формы (см. [7] таблицы 4,6). Для устранения расходимости фактора усиления в точках нулевой кривизны на поверхности медленности в работах [5,10-12] плотность потока фононов усреднялась по площади детектора $\Delta S$. Трудности таких расчетов, а также анализа связи величин коэффициентов усиления с кривизной изоэнергетических поверхностей, как показывают работы [2-5,7-16], обусловлены тем, что, хотя исходные выражения для них были сформулированы почти 50 лет назад, однако аналитического выражения не было получено. Нами решена эта задача: предложен новый метод расчета коэффициента $A^{\lambda}$ и получены простые аналитические выражения для него.

Это позволило проанализировать качественные отличия коэффициентов усиления $A^{\lambda}$ в кубических кристаллах с различным типом анизотропии упругой энергии. Исследована связь величин разрывов коэффициентов усиления поперечных мод c изменениями параметра анизотропии $k-1 \quad\left(k-1=\left(c_{12}+2 c_{44}\right.\right.$ $\left.-c_{11}\right) /\left(c_{11}-c_{44}\right), c_{i j}-$ упругие модули второго порядка). Проанализированы его зависимости от типов кривизны изоэнергетических поверхностей для всех акустических мод в кубических кристаллах с положительной $k-1>0(\mathrm{Fe}, \mathrm{Cu}, \mathrm{MgO}, \mathrm{InSb}, \mathrm{GaAs}, \mathrm{GaN}, \mathrm{Ge}$, $\mathrm{Si}$, алмаз, $\mathrm{LiF})$ и отрицательной $k-1<0\left(\mathrm{CaF}_{2}, \mathrm{SrF}_{2}\right.$ 
и $\mathrm{PbS}$ ) анизотропией упругих модулей второго порядка (см. [17], табл. 1). Так, например, для волновых векторов в плоскости грани куба спектр быстрых поперечных мод в кристаллах первого типа $(k-1>1)$ и медленных поперечных мод в кристаллах второго типа $(k-1<0)$ изотропен, и, казалось бы, коэффициенты усиления для них должны равнятся единице. Однако нами показано, что в полупроводниковых кристаллах первого типа для быстрых поперечных мод преобладают эффекты фокусировки, и их значения больше единицы. Во всех кристаллах второго типа $(k-1<0)$ для медленных поперечных мод преобладают эффекты дефокусировки, и коэффициенты усиления для них оказываются меньше единицы.

\section{2. Коэффициент усиления потока фононов}

Определим выражение для коэффициента $A^{\lambda}$ согласно [2]. Пусть на одной из граней образца находится источник фононов (нагреватель), а на противополжной грани - детектор фононов. Они соединены вектором $\mathbf{R}$. В изотропной среде волны, покидающие источник, будут достигать детектора в случае, если их волновые вектора лежат в телесном угле $\delta \Omega_{q}=\sin \theta d \theta d \varphi \quad((\theta, \varphi)-$ угловые переменные вектора $\mathbf{q})$ в окрестности направления $\mathbf{R}$ (см. рис $1, a)$. В случае анизотропной среды волна c волновым вектором q и поляризацией $\lambda$ достигает центра детектора, если направления групповой скорости лежат в телесном угле $\delta \Omega_{V}$ в окрестности вектора $\mathbf{R}$, т. е. (см. рис. $1, b): \mathbf{V}_{g}^{\lambda} \| \mathbf{R}$. Из рис. 2 видно,что этому условию для поперечных мод может удовлетворять не одна волна, а по крайней мере, две или три волны в направлениях волновых векторов, соответствующих областям с отрицательной кривизной на изоэнергетической поверхности. В работе [2] показано, что коэффициент усиления может быть определен через отношение телесных углов для

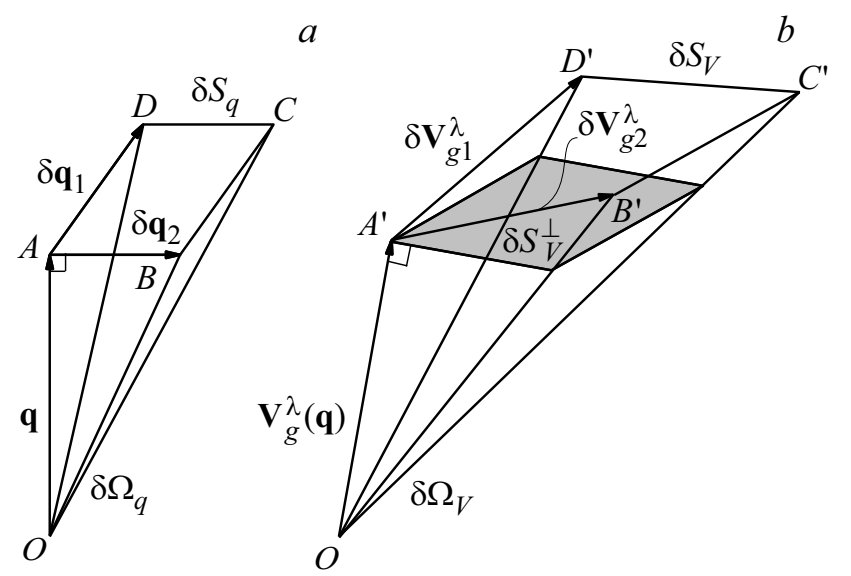

Рис. 1. Схемаичное изображение телесных углов: $(a)-\delta \Omega_{q}$ в q-пространстве и $(b)-\delta \Omega_{V}$ в пространстве групповых скоростей.
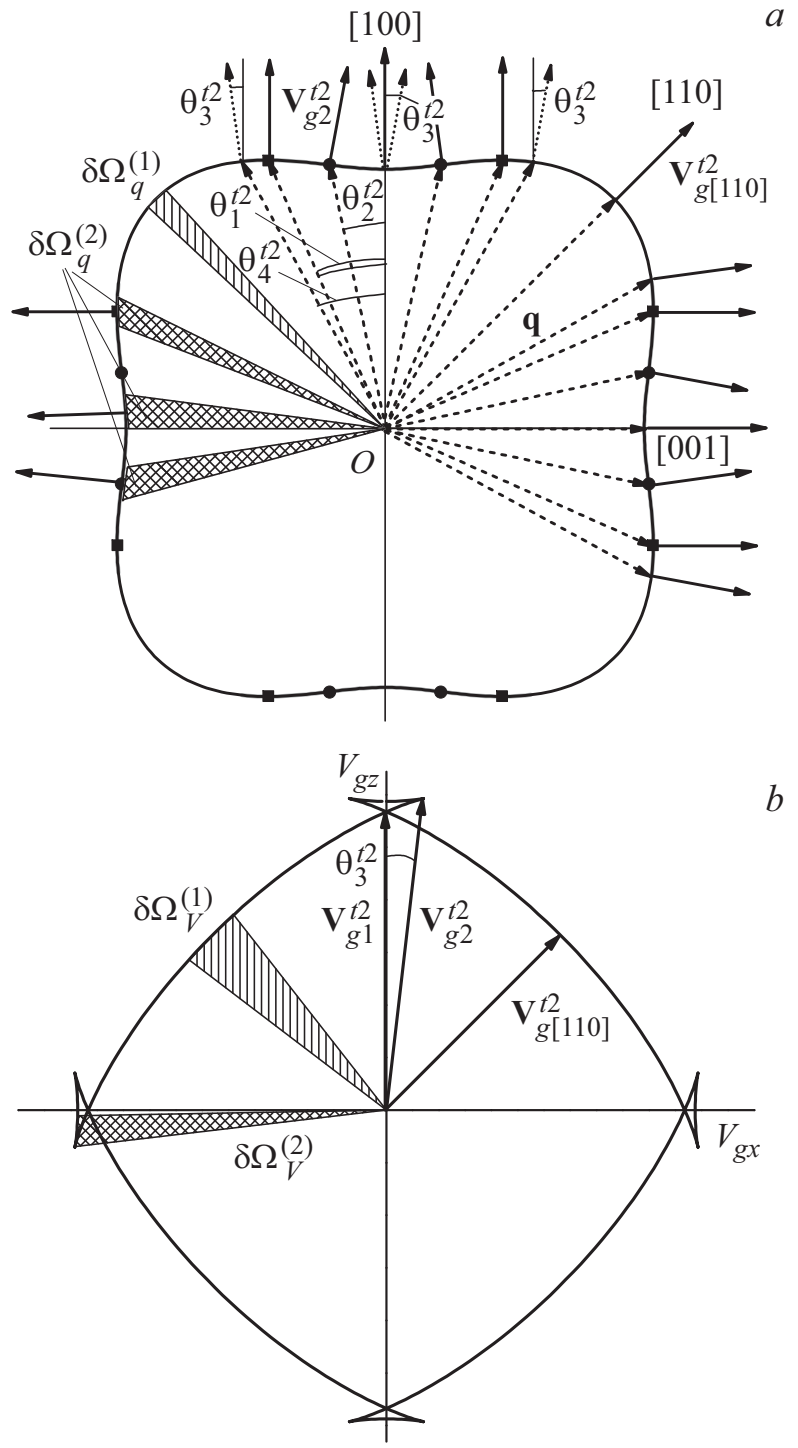

Рис. 2. Сечения изоэнергетической поверхности $(a)$ и волновой поверхности в пространстве групповых скоростей $(b)$ плоскостью грани куба для медленной поперечной моды в кристалле кремния. Значения углов $\theta_{i}^{t 2}$ для плоскости грани куба определены в работе [18]. Вектора групповых скоростей $\mathbf{V}_{g 1}^{t 2}$, $\mathbf{V}_{g 2}^{t 2}$ и $\mathbf{V}_{g[110]}^{t 2}$ на рисунке $(a)$ соответствуют векторам $\mathbf{V}_{g 1}^{t 2}, \mathbf{V}_{g 2}^{t 2}$ и $\mathbf{V}_{g[110]}^{t 2}$ на рисунке $(b)$.

волновых векторов и групповых скоростей

$$
A^{\lambda}(\theta, \varphi)=\delta \Omega_{q} / \delta \Omega_{V}^{\lambda} .
$$

Поскольку распределение волновых векторов, в отличие от групповых скоростей, изотропно в кристалле, то плотность потока фононов будет увеличиваться или уменьшаться на величину $A^{\lambda}(\theta, \varphi)$ по отношению к изотропной среде. Поэтому задача сводится к вычислению отношения телесного угла в q-пространстве к телесному углу в пространстве групповых скоростей. В q-пространстве из точки $O$ под углом $\delta \Omega_{q}$ виден параллелограмм $A B C D$ (см. рис. $1, a$ ). Вектора $\mathbf{q}=q \mathbf{n}$, 
$\delta q_{1}=q \mathbf{n}_{1}$ и $\delta q_{2}=q \mathbf{n}_{2}$ образуют тройку взаимно ортогональных векторов [19]. Тогда для изотропной среды имеем

$$
\delta \Omega_{q}=(\delta q / q)^{2} .
$$

Соответствующий телесный угол для тех же вариаций волновых векторов $\left(\delta \mathbf{q}_{1}\right.$ и $\left.\delta \mathbf{q}_{2}\right)$ в пространстве групповых скоростей $\delta \Omega_{V}$ есть угол, под которым виден параллелограмм $A^{\prime} B^{\prime} C^{\prime} D^{\prime}$ (см. рис. $\left.1, b\right)$, где

$$
\begin{gathered}
\delta V_{q 1 i}^{\lambda}=V_{g i}^{\lambda}\left(\mathbf{q}+\delta \mathbf{q}_{1}\right)-V_{g i}^{\lambda}(\mathbf{q})=\partial V_{g i}^{\lambda} / \partial \mathbf{q} \cdot \delta \mathbf{q}_{1}, \\
\delta V_{q 2 i}^{\lambda}=V_{g i}^{\lambda}\left(\mathbf{q}+\delta \mathbf{q}_{2}\right)-V_{g i}^{\lambda}(\mathbf{q})=\partial V_{g i}^{\lambda} / \partial \mathbf{q} \cdot \delta \mathbf{q}_{2},
\end{gathered}
$$

где $V_{g i}^{\lambda}-i$-ая компонента групповой скорости в ортогональной системе координат. Тогда телесный угол $\delta \Omega_{V}^{\lambda}$ в пространстве групповых скоростей примет вид [2]

$$
\delta \Omega_{V}^{\lambda}=\delta S_{V}^{\perp} /\left(V_{g}^{\lambda}\right)^{2}=\left|\left(\left[\delta \mathbf{V}_{g 1}^{\lambda} \times \delta \mathbf{V}_{g 2}^{\lambda}\right] \cdot \mathbf{V}_{g}^{\lambda}\right) /\left(V_{g}^{\lambda}\right)^{3}\right| .
$$

А коэффициент усиления $A^{\lambda}(\theta, \varphi)$ был представлен в виде [2]

$$
\begin{aligned}
& \left(A^{\lambda}(\theta, \varphi)\right)^{-1}=\frac{\delta \Omega_{V}^{\lambda}}{\delta \Omega_{q}} \\
& \quad=\frac{q^{2}}{\left(V_{g}^{\lambda}\right)^{3}}\left|\left(\left[\left\{\mathbf{n}_{1} \cdot \frac{\partial \mathbf{V}_{g 1}^{\lambda}}{\partial \mathbf{q}}\right\} \times\left\{\mathbf{n}_{2} \cdot \frac{\partial \mathbf{V}_{g 2}^{\lambda}}{\partial \mathbf{q}}\right\}\right] \cdot \mathbf{V}_{g}^{\lambda}\right)\right| .
\end{aligned}
$$

Далее мы покажем, что при вычислении коэффициента $A^{\lambda}(\theta, \varphi)$ следует пользоваться сферической системой координат $\left(\mathbf{n}_{1}=\mathbf{e}_{\theta}, \mathbf{n}_{2}=\mathbf{e}_{\varphi}\right)$. Это позволяет получить для него аналитическое выражение. Вектора групповой скорости и производная $\partial V_{g i}^{\lambda} / \partial \mathbf{q}$ в сферической системе координат могут быть представлены в виде

$$
\begin{gathered}
\mathbf{V}_{g}^{\lambda}(\theta, \varphi)=S^{\lambda}(\theta, \varphi) \tilde{\mathbf{V}}_{g}^{\lambda}(\theta, \varphi), \quad \tilde{\mathbf{V}}_{g}^{\lambda}(\theta, \varphi)=\mathbf{n}+S_{\theta}^{\lambda} \mathbf{e}_{\theta}+S_{\varphi}^{\lambda} \mathbf{e}_{\varphi}, \\
S_{\theta}^{\lambda}(\theta, \varphi)=\left(\frac{1}{S^{\lambda}}\right) \frac{\partial S^{\lambda}}{\partial \theta}, \quad S_{\varphi}^{\lambda}(\theta, \varphi)=\frac{1}{\sin \theta}\left(\frac{1}{S^{\lambda}}\right) \frac{\partial S^{\lambda}}{\partial \varphi}, \quad(6)
\end{gathered}
$$

Здесь фазовая скорость $S^{\lambda}(\theta, \varphi)$ в модели анизотропного континуума определена в работах $[17,20]$, а вектора $\mathbf{n}, \quad \mathbf{e}_{\theta}$ и $\mathbf{e}_{\varphi}$ образуют взаимно ортогональную тройку единичных векторов: $\mathbf{n}=\mathbf{q} / q=$ $=(\sin \theta \cos \varphi, \sin \theta \sin \varphi, \cos \theta)$ - единичный волновой вектор фонона, $\mathbf{e}_{\theta}=(\cos \theta \cos \varphi, \cos \theta \sin \varphi,-\sin \theta), \mathbf{e}_{\varphi}=$ $=(-\sin \varphi, \cos \varphi, 0)$ (см. $[18,20])$. Из формул $(3-4)$ для векторов $\delta \mathbf{V}_{g 1}^{\lambda}$ и $\delta \mathbf{V}_{g 2}^{\lambda}$ в получим

$$
\begin{aligned}
\delta V_{g 1 i}^{\lambda} & =\left(\partial V_{g i}^{\lambda} / \partial \mathbf{q} \cdot \delta \mathbf{q}_{1}\right)=\left(\mathbf{e}_{\theta} \partial V_{g i}^{\lambda} / \partial \mathbf{q}\right) \cdot \delta q \\
& =(\delta q / q) \cdot\left(\partial V_{g i}^{\lambda} \partial \theta\right), \\
\delta V_{g 2 i}^{\lambda} & =\left(\partial V_{g i}^{\lambda} / \partial \mathbf{q} \cdot \delta \mathbf{q}_{2}\right)=\left(\mathbf{e}_{\theta} \partial V_{g i}^{\lambda} / \partial \mathbf{q}\right) \cdot \delta q \\
& =(\delta q / q \sin \theta) \cdot\left(\partial V_{g i}^{\lambda} \partial \varphi\right) .
\end{aligned}
$$

Из формул (3)-(6) получим выражение для $\delta \mathbf{S}_{V}=\left[\delta \mathbf{V}_{g i}^{\lambda} \times \delta \mathbf{V}_{g 2}^{\lambda}\right]$. Как видно из рисунка $1, b$, площадка $\delta \mathbf{S}_{V}$ не ортогональна вектору групповой скорости $\mathbf{V}_{g}^{\lambda}$. Поэтому спроектируем ее на поверхность перпендикулярную единичному вектору групповой скорости: $\quad \delta S_{V}^{\perp}=\left(\delta \mathbf{S}_{V} \cdot \mathbf{V}_{g}^{\lambda}\right) / V_{g}^{\lambda} \quad$ (см. $\quad$ затемненную область на рис. $1, b)$. Тогда коэффициент $A^{\lambda}(\theta, \varphi)$ в сферической системе координат можно представить в виде

$$
\begin{aligned}
\left(A^{\lambda}(\theta, \varphi)\right)^{-1} & =\frac{\partial \Omega_{V}^{\lambda}}{\partial \Omega_{q}} \\
& =\frac{1}{\left(V_{g}^{\lambda}\right)^{3} \sin \theta} \times\left|\left(\left[\frac{\partial \mathbf{V}_{g}^{\lambda}}{\partial \theta} \times \frac{\partial \mathbf{V}_{g}^{\lambda}}{\partial \varphi}\right] \cdot \mathbf{V}_{g}^{\lambda}\right)\right|
\end{aligned}
$$

Использование декартовой системы координат приводит к результату

$$
\begin{aligned}
\left(A^{\lambda}(\theta, \varphi)\right)^{-1}= & \frac{1}{\left(V_{g}^{\lambda}\right)^{3} \sin \theta} \cdot \mid\left(\frac{\partial V_{g y}^{\lambda}}{\partial \theta} \frac{\partial V_{g z}^{\lambda}}{\partial \varphi}-\frac{\partial V_{g z}^{\lambda}}{\partial \theta} \frac{\partial V_{g y}^{\lambda}}{\partial \varphi}\right) V_{g x}^{\lambda} \\
& +\left(\frac{\partial V_{g z}^{\lambda}}{\partial \theta} \frac{\partial V_{g x}^{\lambda}}{\partial \varphi}-\frac{\partial V_{g x}^{\lambda}}{\partial \theta} \frac{\partial V_{g z}^{\lambda}}{\partial \varphi}\right) V_{g y}^{\lambda} \\
& +\left(\frac{\partial V_{g x}^{\lambda}}{\partial \theta} \frac{\partial V_{g y}^{\lambda}}{\partial \varphi}-\frac{\partial V_{g y}^{\lambda}}{\partial \theta} \frac{\partial V_{g x}^{\lambda}}{\partial \varphi}\right) V_{g z}^{\lambda} \mid .
\end{aligned}
$$

Здесь $V_{g x}^{\lambda}(\theta, \varphi), V_{g y}^{\lambda}(\theta, \varphi), V_{g z}^{\lambda}(\theta, \varphi)$ - компоненты групповой скорости фононов с поляризацией $\lambda$ в декартовой системе координат

$$
\begin{aligned}
V_{g x}^{\lambda}(\theta, \varphi)= & S^{\lambda}(\theta, \varphi)\left\{\sin \theta \cos \varphi+S_{\theta}^{\lambda}(\theta, \varphi) \cos \theta \cos \varphi\right. \\
& \left.-S_{\varphi}^{\lambda}(\theta, \varphi) \sin \varphi\right\}, \\
V_{g y}^{\lambda}(\theta, \varphi)= & S^{\lambda}(\theta, \varphi)\left\{\sin \theta \sin \varphi+S_{\theta}^{\lambda}(\theta, \varphi) \cos \theta \sin \varphi\right. \\
& \left.+S_{\varphi}^{\lambda}(\theta, \varphi) \cos \varphi\right\}, \\
V_{g z}^{\lambda}(\theta, \varphi)= & S^{\lambda}(\theta, \varphi)\left\{\cos \theta-S^{\lambda}(\theta, \varphi) \sin \theta\right\} .
\end{aligned}
$$

Выражение (9) для коэффициента усиления при использовании декартовой системы координат содержит более 300 членов и является слишком громоздким для анализа.

В работах $[10,11]$ предложен другой способ вычисления коэффициента усиления через производные углов групповой скорости $\theta_{V}^{\lambda}$ и $\varphi_{V}^{\lambda}$, определяющих направление вектора $\mathbf{V}_{g}^{\lambda}$. Согласно определению (1) $A^{\lambda}(\theta, \varphi)$ можно записать в виде

$$
\left(A^{\lambda}(\theta, \varphi)\right)^{-1}=\frac{\delta \Omega_{V}^{\lambda}}{\delta \Omega_{q}^{\lambda}}=\frac{d\left(\cos \theta_{V}^{\lambda}\right) d \varphi_{V}^{\lambda}}{d(\cos \theta) d \varphi} .
$$

Здесь $\delta \Omega_{q}=d(\cos \theta) d \varphi-$ телесный угол в q-пространстве под которым видна площадка $\left|\delta \mathbf{S}_{q}\right|=\left|\delta \mathbf{q}_{1} \times \mathbf{q}_{2}\right|$, образованная векторами $\delta \mathbf{q}_{1}$ и $\delta \mathbf{q}_{2}$, а $\delta \Omega_{V}^{\lambda}=d\left(\cos \theta_{V}^{\lambda}\right) d \varphi_{V}^{\lambda}-$ соответсвующий ему телесный угол в пространстве 
групповых скоростей под которым видна площадка $\left|\delta \mathbf{S}_{V} \cdot \mathbf{V}_{g}^{\lambda} / V_{g}^{\lambda}\right|$, образованная векторами $\delta \mathbf{V}_{g 1}^{\lambda}$ и $\delta \mathbf{V}_{g 2}^{\lambda}$ (см. рис. 1). Тогда коэффицент усиления можно записать через якобиан перехода от переменных $\left(\theta_{V}^{\lambda}, \varphi_{V}^{\lambda}\right)$ к переменным $(\theta, \varphi)[10,11]$

$$
\begin{aligned}
\left(A^{\lambda}(\theta, \varphi)\right)^{-1} & =\left|\begin{array}{cc}
\partial\left(\cos \theta_{V}^{\lambda}\right) / \partial(\cos \theta) & \partial\left(\cos \theta_{V}^{\lambda}\right) / \partial \varphi \\
\partial \varphi_{V}^{\lambda} / \partial(\cos \theta) & \partial \varphi_{V}^{\lambda} / \partial \varphi
\end{array}\right| \\
& =\frac{1}{\sin \theta}\left|\frac{\partial\left(\cos \theta_{V}^{\lambda}\right)}{\partial \theta} \frac{\partial \varphi_{V}^{\lambda}}{\partial \varphi}-\frac{\partial\left(\cos \theta_{V}^{\lambda}\right)}{\partial \varphi} \frac{\partial \varphi_{V}^{\lambda}}{\partial \theta}\right| .
\end{aligned}
$$

Для нахождения зависимостей $\theta_{V}^{\lambda}(\theta, \varphi)$ и $\varphi_{V}^{\lambda}(\theta, \varphi)$ в работах $[10,11]$ опять использовались выражения для групповой скорости в декартовой системе координат

$$
\mathbf{V}_{g}^{\lambda}=V_{g}^{\lambda}\left(\sin \theta_{V}^{\lambda} \cos _{V}^{\lambda} \mathbf{i}+\sin \theta_{V}^{\lambda} \sin \varphi_{V}^{\lambda} \mathbf{j}+\cos \theta_{V}^{\lambda} \mathbf{k}\right)
$$

В результате получено

$$
\cos \theta_{V}^{\lambda}(\theta, \varphi)=\frac{V_{g z}^{\lambda}(\theta, \varphi)}{V_{g}^{\lambda}(\theta, \varphi)} \text { и } \varphi_{V}^{\lambda}(\theta, \varphi)=\operatorname{arctg} \frac{V_{g y}^{\lambda}(\theta, \varphi)}{V_{g x}^{\lambda}(\theta, \varphi)} .
$$

Хотя формулы (12)-(14) значительно упрощают численный анализ коэффициента усиления, по сравнению с использованием выражений (9)-(10), однако его расчет в декартовой системе координат остается достаточно громоздким. При этом формулы (12)-(14) не дают замкнутого аналитического выражения для коэффициента $A^{\lambda}(\theta, \varphi)$.

Проведенный нами анализ показал, что использование сферической системы координат для векторов групповых скоростей и их производных позволяет получить точное аналитическое решение для коэффициента усиления $A^{\lambda}(\theta, \varphi)$ и провести детальный анализ его особенностей. Для этого воспользуемся формулами (3)-(6) и вычислим скалярное произведение $\left(\left[\partial \mathbf{V}_{g}^{\lambda} / \partial \theta \times \partial \mathbf{V}_{g}^{\lambda} / \partial \varphi\right] \cdot \mathbf{V}_{g}^{\lambda}\right)$ в сферической системе координат. Тогда получим выражение для $A^{\lambda}(\theta, \varphi)$ через угловые компоненты групповой скорости $S_{\theta}^{\lambda}, S_{\varphi}^{\lambda}$ и их производные

$$
\begin{aligned}
A^{\lambda}(\theta, \varphi)= & \left(\tilde{V}_{g}^{\lambda}\right)^{3} \mid\left(1+\left(S_{\theta}^{\lambda}\right)^{2}+\frac{\partial S_{\theta}^{\lambda}}{\partial \theta}\right) \\
& \times\left(1+S_{\theta}^{\lambda} \frac{\cos \theta}{\sin \theta}+\left(S_{\varphi}^{\lambda}\right)^{2}+\frac{1}{\sin \theta} \frac{\partial S_{\varphi}^{\lambda}}{\partial \varphi}\right) \\
& -\left.\left(\frac{1}{\sin \theta} \frac{\partial S_{\theta}^{\lambda}}{\partial \varphi}+S_{\varphi}^{\lambda}\left(S_{\theta}^{\lambda}-\frac{\cos \theta}{\sin \theta}\right)\right)^{2}\right|^{-1}
\end{aligned}
$$

Очевидно, что для модели изотропной среды угловые производные $S_{\theta}^{\lambda}, S_{\varphi}^{\lambda}$ равны нулю, а коэффициент усиления равен единице. Для дальнейшего анализа особенностей распространения потока фононов в упруго анизотропных кристаллах мы будем пользоваться аналитическим выражением (15).

Спектр упругих волн и групповые скорости реальных кристаллов имеют достаточно сложный вид. Чтобы наглядно представить телесный угол $\delta \Omega_{q}$ в $\mathbf{q}$-пространстве и соответствующий ему угол $\delta \Omega_{V}$ в пространстве групповых скоростей, обратимся к рис. 2. На нем изображены сечения изоэнергетической поверхности и волновой поверхности в пространстве групповых скоростей плоскостью грани куба для медленной поперечной моды в кристалле кремния (рис. 2). Волновая поверхность - это геометрическое место концов векторов групповой скорости для всевозможных направлений волновых векторов в кристалле [21]. Согласно ([21] см. уравнение $(24.13))$, ее можно определить выражением

$$
\left(\mathbf{n} \cdot V_{g}^{\lambda}\right) / S^{\lambda}(\theta, \varphi)=1
$$

Волновую поверхность можно также задать параметрически, взяв в качестве праметров углы волнового вектора $\theta$ и $\varphi$. Для сечения $\{010\}$ она имеет вид

$$
\left\{\begin{array}{l}
V_{g x}^{\lambda}=V_{g x}^{\lambda}(\theta, 0), \\
V_{g y}^{\lambda}=0, \\
V_{g z}^{\lambda}=V_{g z}^{\lambda}(\theta, 0) .
\end{array}\right.
$$

Здесь компоненты групповой скорости $\mathbf{V}_{g}^{\lambda}(\theta, \varphi)$ определены формулами (10). В окрестности направления [110] телесный угол $\delta \Omega_{V}^{(1)}$ соответствует углу $\delta \Omega_{q}^{(1)}$ (см. рис. 2). В этом случае непосредственный расчет дает значения коэффициент $A_{[110]}^{t 2}<1$, т. е. $\delta \Omega_{V}^{(1)}>\delta \Omega_{q}^{(1)}$, и медленная поперечная мода для этого направления дефокусируется (см. рис. 2 и 3 ).

В окрестности направления [100] телесный угол $\delta \Omega_{V}^{(2)}$ (см. рис. $2, b$ ) соответствует трем различным значениям телесного угла $\delta \Omega_{q}^{(2)}$ (см. рис. 2, $a$ ). Поэтому полное отношение $\delta \Omega_{q} / \delta \Omega_{V}$ для направления [100] будет гораздо больше единицы, что указывает на большое усиление потока фононов в окрестности направления фокусировки. Рассмотрим более подробно изоэнергетическую поверхность для моды $t_{2}$. В работе [18] показано, что в окрестности направлений [100] в интервале углов $-\theta_{1} \leq \theta \leq \theta_{1}$ функция $\theta_{g}^{t 2}(\theta)<0$, а при $\theta_{2}=0.21$ она имеет локальный минимум. Углы $\pm \theta_{2}$ определяют направления векторов групповой скорости $\mathbf{V}_{g 2}^{t 2}$ на рис. $2, b$ к границам складок на волновой поверхности в пространстве групповых скоростей. Эти же углы определяют направления волновых векторов к точкам нулевой кривизны на изоэнергетической поверхности [18]. В этих точках происходит переход от выпуклых к вогнутым областям, и кривизна обращается в ноль. Положения точек нулевой кривизны обозначены кружочками на рис. 2. В трехмерном случае эти точки образуют линии нулевой кривизны на изоэнергетической поверхности. Поскольку фононный поток ортогонален этой поверхности и обратно пропорционален ее кривизне в данной точке [2-4], то 


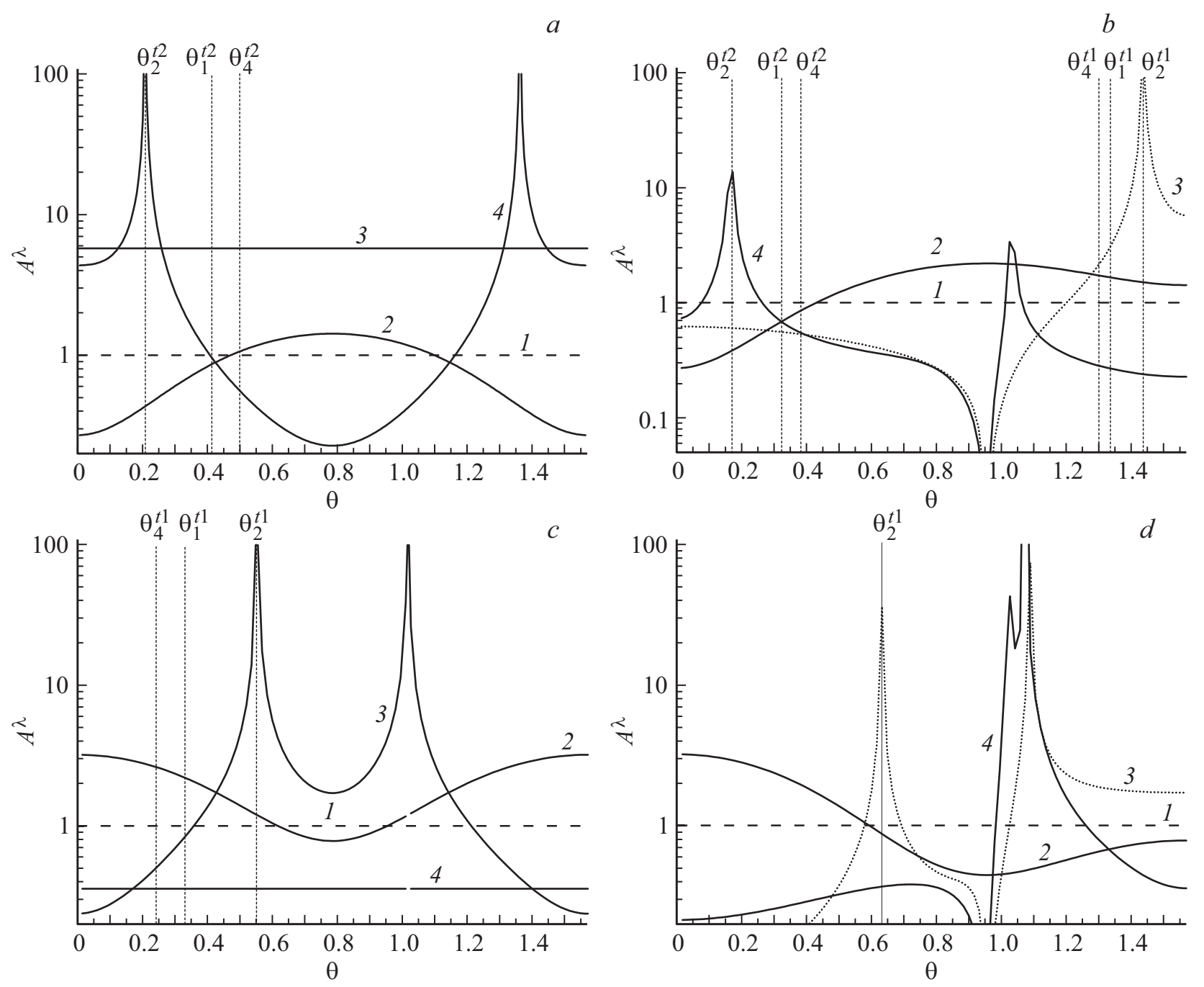

Рис. 3. Угловые зависимости коэффициентов усиления $A^{\lambda}(\theta, \varphi)$ в кристаллах $\mathrm{Si}(a),(b)$ и $\mathrm{CaF}_{2}(c),(d)$ для волновых векторов в плоскости грани куба $(a),(c)$ и диагональной плоскости $(b),(d)$. Штриховые линии 1 соответствуют модели изотропной среды, кривые 2 - продольным фононам, кривые 3 - быстрой поперечной моде $t_{1}$, кривые 4 - медленной поперечной моде $t_{2}$.

точки нулевой кривизны математически соответствуют бесконечному потоку фононов от точечного источника тепла вдоль соответствующего направления групповой скорости.

\section{3. Анализ угловых зависимостей коэффициента усиления}

Воспользуемся (15) и проанализируем угловые зависимости коэффициента усиления в кристаллах кремния (см. рис. 3,a). В модели изотропной среды коэффициент $A^{\lambda}(\theta, 0)=1$. Поэтому интервалы углов, в которых выполняется неравенство $A^{\lambda}(\theta, 0)>1$, можно отнести к области фокусировки фононов, а интервалы, в которых выполняется обратное неравенство - к области дефокусировки фононов. Очевидно, что в точках нулевой кривизны $\left(\theta=\theta_{2}^{t 2}\right)$ на изоэнергетической поверхности коэффициент усиления поперечной моды $t_{2}$ обращается в бесконечность (см. рис. 3, $a$, кривая 4). Минимального значения он достигает в направлении дефокусировки [110]: для $\mathrm{Si} A_{[110]}^{t 2}=0.23$. Продольные фононы фокусируются и дефокусируются в направлениях [111] и [100] соответственно, и в этих случаях для $\mathrm{Si} A_{[111]}^{L}=2.20$ и $A_{[100]}^{L}=0.27$. В направлении [110] для них имеет место локальный максимум фокусировки, и $A_{[110]}^{L}=1.42$. $\mathrm{B}$ кристалле второго типа $\mathrm{CaF}_{2}$ коэффициент усиления быстрой поперечной моды в точках нулевой кривизны $\left(\right.$ при $\left.\theta=\theta_{2}^{(t 1)}\right)$ на изоэнергетической поверхности обращается в бесконечность (см. рис. $3, b$, кривая 3 ). Для продольных фононов в направлении [100] имеет место фокусировка, и коэффициент усиления $A_{[110]}^{L}=3.21$, а в направлении [110] они дефокусируются, и коэффициент $A_{[110]}^{L}=0.78$.

Проведенный нами анализ коэффициентов усиления для быстрой поперечной моды в кристаллах первого и медленной поперечной моды в кристаллах второго типа 
позволил обнаружить интересную особенность. Дело в том, что для волновых векторов в плоскости грани куба спектр обеих поперечных мод в кубических кристаллах является изотропным (см. рис. 1 работы [17]), и следовало ожидать, что коэффициент $A^{\lambda}(\theta, 0)$ будет равен единице, как и в модели изотропной среды. Однако непосредственный расчет с использованием выражений (15) дал для него величину, которая в кристаллах кремния значительно больше единицы $-A^{t 1}=5.74$ (см. рис. $3, a$, кривая 3), а для кристалла второго типа $\mathrm{CaF}_{2}$ заметно меньше единицы - (см. рис. 3, $c$, кривая 4). Поскольку ранее на эту особенность коэффициента усиления не обращалось внимание, то мы проанализируем ее для кристаллов с различным типом анизотропии упругой энергии более детально. Для этого разложим фазовую скорость быстрой поперечной моды в кристаллах первого типа из уравнения (4) работы [17] вблизи плоскости грани куба при $\varphi \ll 1$

$$
\begin{gathered}
S^{t 1}(\theta, \varphi) \approx \sqrt{\frac{c_{44}}{\rho}}\left(1-\frac{\delta}{2} \varphi^{2} \sin ^{2} \theta\right), \\
\delta=\left[\frac{c_{11}-c_{44}}{c_{44}}\right] \frac{(k-1)(2 k+1)}{(k+1)} .
\end{gathered}
$$

Из $(18)$ видно, что в кристаллах первого типа $S^{t 1}(\theta, 0)$ имеет максимальное значение. Из выражения (15) в случае $\varphi \rightarrow 0$ следует, что вклад в коэффициент усиления моды $t_{1}$ дает только вторая производная фазовой скорости $\partial^{2} S^{t 1} / \partial \varphi^{2}$

$$
A^{t 1}(\theta, 0)=\left|1+\frac{1}{\sin ^{2} \theta} \frac{\partial^{2} S^{t 1}}{\partial \varphi^{2}} \sqrt{\frac{\rho}{c_{44}}}\right|^{-1}=\left|\frac{1}{1-\delta}\right| .
$$

Для больщинства полупроводниковых кристаллов первого типа значения параметра $\delta$ лежит в интервале $0 \leq \delta \leq 2$, и коэффициент усиления быстрой поперечной моды может значительно превосходить единицу. Однако для металлических кристаллов первого типа $\mathrm{Na}, \mathrm{K}, \mathrm{Li}$ и $\mathrm{Cu}$ параметр $\delta>2$, и коэффициент усиления быстрой поперечной моды оказывается меньшим единицы (см. табл. 1). Как видно из табл. 1, корреляция между значениями коэффициента $A^{t 1}(\theta, 0)$ и величинами параметра анизотропии $(k-1)$ отсутствует. Так, например, для кристаллов YAG параметр анизотропии мал $(k-1 \approx 0.03)$, и коэффициент усиления близок к единице: $A^{t 1}(\theta, 0)=1.08$. Для кристаллов $\mathrm{HgSe}$ параметр анизотропии в 20 раз больше, однако коэффициент усиления $A^{t 1}(\theta, 0)=1.02$ оказывается меньше, чем для кристаллов YAG. Для кристаллов $\mathrm{GaN}$ параметр $k-1$ имеет максимальное значение, однако коэффициент усиления оказывается таким же, как для кристаллов YAG (см. табл. 1). Из рассмотренных кристаллов первого типа коэффициент усиления достигает максимального значения для $\mathrm{MgO}\left(A^{t 1}(\theta, 0) \approx 16.11\right)$, у которого параметр анизотропии $k-1=0.7$ мало отличается от значения для $\mathrm{HgSe}$ (см. табл. 1). Минимальное значение коэффициент $A^{t 1}(\theta, 0)=0.4$ достигает для щелочного
Таблица 1. Значения параметров $k-1, \delta$ и коэффициентов усиления $A^{t 1}(\theta, 0)$ для быстрой поперечной моды в кубических кристаллах первого типа

\begin{tabular}{c|l|l|l}
\hline Кристалл & $k-1$ & $\delta$ & $A^{t 1}$ \\
\hline $\mathrm{Fe}$ & 1.15 & 1.91 & 1.09 \\
$\mathrm{Cu}$ & 1.12 & 2.31 & 0.76 \\
$\mathrm{GaAs}$ & 0.9 & 1.49 & 2.04 \\
$\mathrm{InSb}$ & 0.81 & 1.63 & 1.58 \\
$\mathrm{MgO}$ & 0.7 & 1.06 & 16.11 \\
$\mathrm{GaSb}$ & 0.85 & 1.47 & 2.14 \\
$\mathrm{Ge}$ & 0.87 & 1.32 & 3.13 \\
$\mathrm{GaN}$ & 1.28 & 1.92 & 1.08 \\
$\mathrm{LiF}$ & 0.78 & 1.2 & 4.89 \\
$\mathrm{Si}$ & 0.67 & 1.17 & 5.74 \\
$\mathrm{HgSe}$ & 0.61 & 1.98 & 1.02 \\
$\mathrm{Aлмаз}$ & 0.4 & 0.55 & 2.22 \\
$\mathrm{YAG}$ & 0.03 & 0.076 & 1.08 \\
$\mathrm{Na}$ & 45.1 & 3.47 & 0.40 \\
$\mathrm{Li}$ & 4.83 & 3.31 & 0.43 \\
$\mathrm{~K}$ & 2.28 & 2.98 & 0.51
\end{tabular}

Таблица 2. Значения параметров $k-1, \delta$ и коэффициентов усиления $A^{t 2}$ в кристаллах второго типа для медленной поперечной моды и волновых векторов в плоскости грани куба

\begin{tabular}{c|c|c|c|c|c|c}
\hline Кристалл & $\mathrm{KCl}$ & \multicolumn{1}{|c|}{$\mathrm{NaCl}$} & \multicolumn{1}{c|}{$\mathrm{PbS}$} & $\mathrm{CaF}_{2}$ & \multicolumn{1}{c|}{$\mathrm{SrF}_{2}$} & \multicolumn{1}{c}{$\mathrm{YIG}$} \\
\hline$k-1$ & -0.63 & -0.48 & -0.466 & -0.33 & -0.204 & -0.04 \\
$\delta$ & -4.29 & -2.12 & -2.59 & -1.80 & -0.88 & -0.17 \\
$A^{22}$ & 0.19 & 0.320 & 0.28 & 0.36 & 0.53 & 0.86
\end{tabular}

металла $\mathrm{Na}$, у которого параметр анизотропии упругой энергии имеет максимальное значение: $k-1=45.1$.

В кристаллах второго типа $(k-1<0)$ для волновых векторов в плоскости грани куба фазовая скорость медленной поперечной моды $t_{2}$, принимает минимальные значения. Для нее спектр фононов при $\varphi \ll 1$ определяется уравнением (18), и коэффициент усиления $A^{t 2}(\theta, 0)$ совпадает с выражением (19). Поскольку во всех кристаллах второго типа параметр $\delta<0$, то коэффициент усиления для них оказывается меньше единицы (см. табл. 2 и рис. 3). Как видно из табл. 2, для кристаллов второго типа величины коэффициента усиления обратно пропорциональны параметру анизотропии. Максимальное значение коэффициент усиления $A_{[100]}^{t 2} \approx 0.86$ принимает для кристалла YIG, у которого параметр анизотропии имеет минимальное значение: $k-1=-0.04$. Минимальное значение коэффициент усиления $A_{[100]}^{t 2} \approx 0.19$ достигает для наиболее анизотропного кристалла $\mathrm{KCl}$ с параметром анизотропии $k-1=-0.63$. Таким образом, в коэффициентах усиления медленной поперечной моды для всех кристаллов второго типа преобладают эффекты дефокусировки фононов, поэтому для волновых векторов в плоскости грани куба они меньше единицы. 
Из рис. 3 видно, что коэффициент усиления медленной поперечной моды при $\theta \rightarrow 0$ в окрестности направления [100] претерпевает конечный разрыв. Для кристаллов $\mathrm{Si}$ этот коэффициент при $\theta \rightarrow 0$ имеет различные значения при $\varphi=0$ и $\pi / 4$ : при $\varphi=0$ он оказывается значительно больше единицы $A^{t 2}(0,0) \approx 4.4$, а при $\pi / 4-$ меньше единицы $A^{t 2}(0, \pi / 4)=0.72$. Этот результат оставался физически странным и до сих пор не был объяснен. Как видно из рис. 1 работы [18], в направлении [100] медленная поперечная мода фокусируется, как в плоскости грани куба $(\varphi=0)$, так и в диагональной плоскости $(\pi / 4)$. Поэтому коэффициент усиления в обоих случаях дожен превышать единицу. Для объяснения этого парадокса требуется детальный анализ поведения коэффициента $A^{t 2}(\theta, \varphi)$, исходя из формул (15), при малых значениях угла $\theta$. Дело в том, что при анализе плотности фононных состояний и рассмотрении сечений изоэнергетической поверхности для $\varphi=0$ и $\pi / 4$ в работе [18], мы зафиксировали угол $\varphi$ и учитывали только производные фазовой скорости по $\theta$. Тогда как при вычислении коэффициента $A^{\lambda}(\theta, \varphi)$ учитываются производные по обоим углам. Коэффициент усиления характеризует изоэнергетическую поверхность и обратно пропорционален ее гауссовой кривизне $A \sim 1 / K[11]$. В зависимости от ее кривизны возможны различные типы особенностей: $(a)$ типа „седло“, $(b)$ типа „вогнутый лепесток“ [11]. Тип особенностей на трехмерной поверхности определяется гауссовой кривизной $K$ [22]. Она может быть представлена в виде произведения главных кривизн поверхности: $K=K_{1} K_{2}$. В работе [11] показано, что при $K<0$ ( $K_{1}$ и $K_{2}$ имеют разные знаки) поверхность имеет особенность типа седловой точки; если оба значения $K_{1}$ и $K_{2}$ отрицательны, то поверхность вогнутая („вогнутый лепесток“); если оба значения $K_{1}$ и $K_{2}$ положительны, то поверхность выпуклая.

Для того чтобы проанализировать тип возникающих особенностей в окрестности направления [100] для различных значений угла $\varphi$, разложим выражение для фазовой скорости медленной моды $t_{2}$ при $\theta \ll 1$. Тогда получим

$$
\begin{gathered}
S^{t 2}(\theta, \varphi) \approx \sqrt{\frac{c_{44}}{\rho}}\left(1-\theta^{2} \Delta_{t 2}(\varphi)\right), \\
\Delta_{t 2}(\varphi)=\frac{c_{11}-c_{44}}{4 c_{44}}(k-1) \\
\times\left[\sqrt{(1+2 k) \cos ^{2} 2 \varphi+k^{2}}+k+1\right] .
\end{gathered}
$$

В кристаллах первого типа фазовая скорость медленных поперечных фононов в направлении [100] имеет абсолютный максимум. Для плоскости грани куба вторые производные по $\theta$ и $\varphi$ имеют разные знаки $\left(\partial^{2} S^{t 2} / \partial \theta^{2}<0, \partial^{2} S^{t 2} / \partial \varphi^{2}>0\right)$, поэтому в окрестности грани куба при в кристаллах $\mathrm{Si}$ реализуется особенность типа седловой точки. Для диагональной плоскости вторая производная $\partial^{2} S^{2} / \partial \theta^{2}$ остается отрицательной, а $\partial^{2} S^{t 2} / \partial \varphi^{2}$ становится отрицательной. Поэтому в окрестности диагональной плоскости поверхность становится вогнутой („вогнутый лепесток“).

В предельном случае $\theta \ll 1$ из выражения (15) следует, что зависимость коэффициента усиления медленной поперечной моды от угла $\varphi$, которую при $\varphi=0$ и $\varphi=\pi / 4$ можно представить в виде произведения двух множителей

$$
A^{t 2}\left(0^{+}, \varphi\right)=\frac{1}{\left|1-2 \Delta_{t 2}(\varphi)\right|} \frac{1}{\left|1-2 \Delta_{t 2}(\varphi)-\Delta_{t 2}^{\prime \prime} \varphi\right|},
$$

где

$$
\begin{aligned}
& \Delta_{t 2}^{\prime \prime}(\varphi)=\frac{d^{2} \Delta_{t 2}(\varphi)}{d \varphi^{2}}=-\frac{c_{11}-c_{44}}{4 c_{44}}(k-1)(1+2 k) \\
& \times\left\{\frac{4 \cos 4 \varphi}{\sqrt{(1+2 k) \cos ^{2} 2 \varphi+k^{2}}}+\frac{(1+2 k) \sin ^{2} 4 \varphi}{\left(\sqrt{(1+2 k) \cos ^{2} 2 \varphi+k^{2}}\right)^{3}}\right\} .
\end{aligned}
$$

Для кристаллов $\mathrm{Si}$ при углах $\varphi=0$ и $\pi / 4$ имеем:

$$
\begin{gathered}
\Delta_{t 2}(0)=\frac{c_{11}-c_{44}}{2 c_{44}}\left(k^{2}-1\right)=0.96, \\
\left(1-2 \Delta_{t 2}(0)\right)^{-1}=-1.09 \\
\Delta_{t 2}^{\prime \prime}(0)=-\frac{c_{11}-c_{44}}{c_{44}} \frac{(k-1)(1+2 k)}{(k+1)}=-1.17, \\
\left(1-2 \Delta_{t 2}(0)-\Delta^{\prime \prime}(0)\right)^{-1}=4, \\
\Delta_{t 2}(\pi / 4)=\frac{c_{11}-c_{44}}{4 c_{44}}(k-1)(2 k+1)=0.78, \\
\left(1-2 \Delta_{t 2}(\pi / 4)\right)^{-1}=-1.79, \\
\Delta_{t 2}^{\prime \prime}(\pi / 4)=\frac{c_{11}-c_{44}}{c_{44}} \frac{(k-1)(1+2 k)}{k}=1.88 \\
\left(1-2 \Delta_{t 2}(\pi / 4)-\Delta_{t 2}^{\prime \prime}(\pi / 4)\right)^{-1}=-0.41 .
\end{gathered}
$$

Из приведенных выше значений следует: $A^{t 2}(0.0)=4.36$ и $A^{t 2}(0, \pi / 4)=0.72$. Как видно из оценок $(22)$, главную роль в выполнении неравенств $A^{t 2}(0,0)>1$, а $A^{t 2}(0, \pi / 4)<1$ играет их зависимость от значения производной $\partial^{2} S^{t 2} / \partial \varphi^{2}$. За счет изменения параметpa $\Delta_{t 2}^{\prime \prime}(\varphi)$ множители || $1-2 \Delta_{t 2}(0)|-| \Delta_{t 2}^{\prime \prime}(0)||^{-1}=4$ и $\left(\left|1-2 \Delta_{t 2}(\pi / 4)\right|+\left|\Delta_{t 2}^{\prime \prime}(\pi / 4)\right|\right)^{-1}=0.41$, входящие в коэффициенты $A^{t 2}(0,0)$ и $A^{t 2}(0, \pi / 4)$, отличаются друг от друга на порядок величины. При этом величины $\left|1-2 \Delta_{t 2}(\varphi)\right|$ при переходе от плоскости грани куба к диагональной плоскости изменяются не столь существенно. Из (21) следует, что для плоскостей $\varphi=0$ и $\varphi=\pi / 4$

$$
\begin{gathered}
K_{1}^{t 2}(0, \varphi)=S_{[100]}^{t} \tilde{K}_{1}^{2}(0, \varphi), \tilde{K}_{1}^{t 2}(0, \varphi)=1-2 \Delta_{t 2}(\varphi), \\
K_{2}^{t 2}(0, \varphi)=S_{[100]}^{t} \tilde{K}_{2}^{2}(0, \varphi), \\
\tilde{K}_{2}^{t 2}(0, \varphi)=1-2 \Delta_{t 2}(\varphi)-\Delta_{t 2}^{\prime \prime}(\varphi) .
\end{gathered}
$$

Для кристаллов первого типа с малой упругой анизотропией (таких, как YAG) изоэнергетическая поверхность 


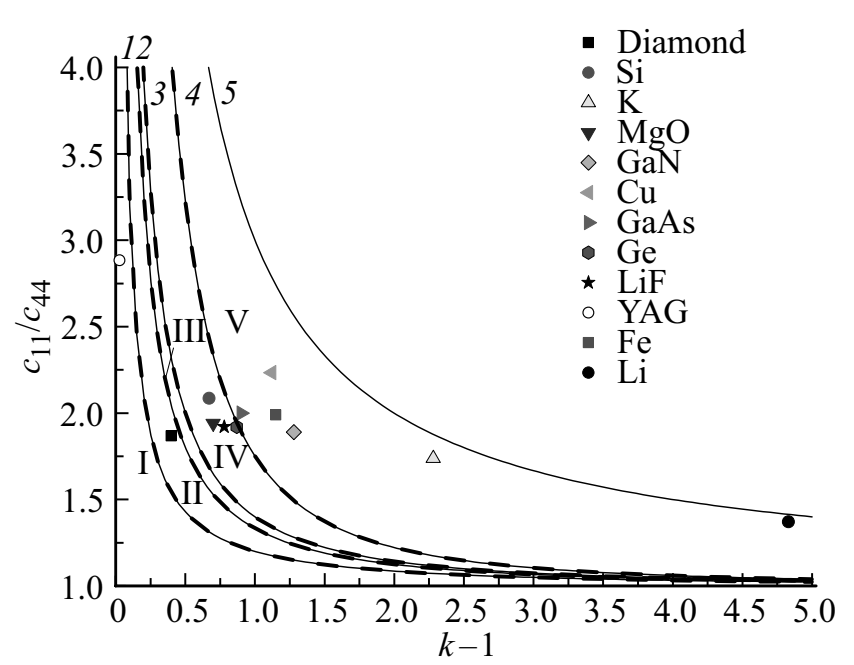

Рис. 4. Графики зависимости $c_{11} / c_{44}$ от параметра $k-1$, определяющие пять областей с различной кривизной изоэнергетических поверхностей для медленных поперечных фононов в кубических кристаллах первого типа. Области I, II, III, $\mathrm{IV}, \mathrm{V}$ ограничены кривыми $1,2,3,4,5$, уравнения для которых задаются неравенствами (24), (26), (27), (28) и (29) соответственно. Сплошные кривые - результаты нашего анализа, штриховые кривые - результаты расчета Эвери [11]. Символами обозначены значения параметров для кристаллов первого типа.

моды $t_{2}$ является всюду выпуклой, а обе главные кривизны поверхности $K_{1}$ и $K_{2}$ положительны (см. табл. 3 ). Из условий $K_{1}>0, K_{2}>0$ и выражений (22) следуют неравенства в которой изоэнергетическая поверхность остается выпуклой (см. рис. 4)

$$
1<\frac{c_{11}}{c_{44}}<1+\frac{2 k}{(k-1)(k+2)(2 k+1)} .
$$

Как следует из (24), эта область ограничена кривой 1

$$
c_{11} / c_{44}=1+2 k /\{(k-1)(k+2)(2 k+1)\} .
$$

Следует отметить, что для всех кристаллов второго типа $(k-1 \leq 0)$ величины $K_{1}$ и $K_{2}$ положительны. Поэтому поверхности постоянной энергии поперечных фононов для них при $\theta \ll 1$ являются выпуклыми (см. табл. 3 ).

С увеличением параметра анизотропии $(k-1)$ мы пересекаем кривую 1 и попадаем во вторую область, для которой при $\theta \ll 1$ в окрестности плоскости грани куба поверхность постоянной энергии медленных поперечных фононов является выпуклой $\left(K_{1}>0\right.$ и $\left.K_{2}>0\right)$, a в окрестности диагональной плоскости реализуется особенность типа седловой точки: $\left(K_{1}>0, K_{2}<0\right.$, см. табл. 3). Из системы неравенств $K_{1}(0,0)>0$, $K_{2}(0,0)>0$ при $\varphi=0$ и $K_{1}(0, \pi / 4)>0, K_{2}(0, \pi / 4)<0$ при $\varphi=\pi / 4$ для второй области получим

$$
1+\frac{2 k}{(k-1)(k+2)(2 k+1)}<\frac{c_{11}}{c_{44}}<1+\frac{1}{k^{2}-1} .
$$

Она ограничена кривыми 1 и 2, последняя определяется выражением $c_{11} / c_{44}=1+1 /\left(k^{2}-1\right)$. Из рассмотренных кристаллов в эту область параметров попадает алмаз (см. рис. 4). В третьей области главные кривизны имеют разные знаки в обеих плоскостях: в плоскости грани куба $K_{1}<0, K_{2}>0$, а в диагональной плоскости $K_{1}>0, K_{2}<0$. Поэтому для обеих плоскостей на изоэнергетической поверхности образуются особенности типа седловых точек. Эта область расположена между кривыми 2 и 3 и определяется неравенствами

$$
1+\frac{1}{k^{2}-1}<\frac{c_{11}}{c_{44}}<1+\frac{2}{(k-1)(2 k+1)} .
$$

Однако ни один из рассмотренных в табл. 3 кристаллов в эту область не попал.

Дальнейшее увеличение параметра анизотропии $(k-1)$ приводит к появлению следующих особенностей на изоэнергетической поверхности при $\theta \ll 1$ : в окрестности плоскости грани куба для моды $t_{2}$ появляется особенность типа седловой точки $\left(K_{1}(0,0)<0\right.$, $\left.K_{2}(0,0)>0\right)$, а в окрестности диагональной плоскости изоэнергетическая поверхность становится вогнутой $\left(K_{1}(0, \pi / 4)<0, K_{2}(0, \pi / 4)<0\right)$. Эти неравенства определяют четвертую область, которая ограничена кривыми 3 и 4 (см. рис. 4)

$$
1+\frac{2}{(k-1)(2 k+1)}<\frac{c_{11}}{c_{44}}<1+\frac{k+1}{k^{2}(k-1)} .
$$

В нее входят такие кристаллы, как $\mathrm{Si}, \mathrm{MgO}, \mathrm{Ge}, \mathrm{LiF}$ (см. рис. 4). При дальнейшем увеличении параметра анизотропии $k-1$ мы пересекаем кривую (4) и попадаем в пятую область, в которой при $\theta \ll 1$ поверхность постоянной энергии для моды $t_{2}$ становится вогнутой для произвольного значения угла $\varphi$ : обе кривизны $K_{1}$ и $K_{2}$ становятся отрицательными (см. табл. 3). Из выражений (22) и условия $c_{11}>c_{12}$ для пятой области получаем соотношения, определяющие кривые 4 и 5

$$
1+\frac{k+1}{k^{2}(k-1)}<\frac{c_{11}}{c_{44}}<1+\frac{2}{k-1} .
$$

В пятую область попали кристаллы $\mathrm{Fe}, \mathrm{Cu}, \mathrm{K}, \mathrm{Li}, \mathrm{Na}$, $\mathrm{GaN}$ c максимальной анизотропией упругой энергией (см. рис. 4).

В работе [11] Эвери провел численный анализ кривизны изоэнергетических поверхностей для кубических кристаллов, исходя из формул (12)-(14), в координатах $c_{11} / c_{44}$ и $c_{12} / c_{44}$ без введения параметра анизотропии $k-1$. Он получил соотношения для модулей упругости, которые разделяют кристаллы с различным типом изоэнергетических поверхностей. Как видно из рис. 4, в координатах $c_{11} / c_{44}$ и $k-1$ наши результаты (сплошные линии), согласуются с анализом, проведенным в [11] (штриховые линии).

В отличие от анализа [11] основное внимание нами уделено исследованию связи величин разрывов коэффициентов усиления поперечных мод в окрестности 
Таблица 3. Значения коэффициентов усиления $A^{t 2}\left(0^{+}, \varphi\right)$ и $A^{t 1}\left(0^{+}, \varphi\right)$ и параметров, характеризующих кривизну изоэнергетических поверхностей для кристаллов первого и второго типа при $\theta \ll 1$

\begin{tabular}{|c|c|c|c|c|c|c|c|}
\hline Кристалл & $c_{11} / c_{44}$ & $1 / \tilde{K}_{1}^{t 2}(0,0)$ & $1 / \tilde{K}_{2}^{t 2}(0,0)$ & $1 / \tilde{K}_{1}^{t 2}(0, \pi / 4)$ & $1 / \tilde{K}_{2}^{t 2}(0, \pi / 4)$ & $A^{t 2}(0,0)$ & $A^{t 2}(0, \pi / 4)$ \\
\hline $\mathrm{Fe}$ & 1.99 & -0.39 & -1.49 & 0.50 & -0.21 & 0.57 & 0.10 \\
\hline $\mathrm{Cu}$ & 2.23 & -0.3 & -1.03 & -0.38 & -0.17 & 0.31 & 0.064 \\
\hline GaAs & 2.00 & -0.62 & -8.31 & -0.86 & -0.29 & 5.16 & 0.25 \\
\hline $\mathrm{InSb}$ & 2.23 & -0.56 & -6.37 & -0.77 & -0.26 & 3.56 & 0.20 \\
\hline $\mathrm{MgO}$ & 1.94 & -1.32 & 3.26 & -2.33 & -0.47 & 4.32 & 1.10 \\
\hline $\mathrm{Ge}$ & 1.92 & -0.77 & 50 & -1.11 & -0.34 & 38.5 & 0.38 \\
\hline $\mathrm{LiF}$ & 1.92 & -0.94 & 7.14 & -1.47 & -0.39 & 6.74 & 0.58 \\
\hline $\mathrm{Na}$ & 1.04 & -0.01 & -0.01 & -0.01 & -0.01 & 0.0002 & 0.0002 \\
\hline $\mathrm{Li}$ & 1.37 & -0.09 & -0.13 & -0.1 & -0.07 & 0.011 & 0.007 \\
\hline K & 1.74 & -0.16 & -0.31 & -0.19 & -0.11 & 0.05 & 0.02 \\
\hline $\mathrm{GaN}$ & 1.89 & -0.37 & -1.25 & -0.47 & -0.2 & 0.46 & 0.096 \\
\hline $\mathrm{Si}$ & 2.09 & -1.09 & 4.0 & -1.79 & -0.41 & 4.36 & 0.72 \\
\hline Алмаз & 1.87 & 6.25 & 1.41 & 2.94 & -1.67 & 8.80 & 4.90 \\
\hline $\mathrm{YaG}$ & 2.89 & 1.11 & 1.03 & 1.08 & 1.29 & 1.14 & 1.40 \\
\hline Кристалл & $c_{11} / c_{44}$ & $1 / \tilde{K}_{1}^{t 1}(0,0)$ & $1 / \tilde{K}_{2}^{t 1}(0,0)$ & $1 / \tilde{K}_{1}^{t 1}(0, \pi / 4)$ & $1 / \tilde{K}_{2}^{t 1}(0, \pi / 4)$ & $A^{t 1}(0,0)$ & $A^{t 1}(0, \pi / 4)$ \\
\hline YIG & 3.52 & 0.84 & 0.95 & 0.87 & 0.69 & 0.8 & 0.6 \\
\hline $\mathrm{SrF}_{2}$ & 4.0 & 0.48 & 0.82 & 0.56 & 0.26 & 0.39 & 0.15 \\
\hline $\mathrm{CaF}_{2}$ & 4.74 & 0.33 & 0.75 & 0.41 & 0.15 & 0.25 & 0.06 \\
\hline $\mathrm{PbS}$ & 5.12 & 0.25 & 0.74 & 0.33 & 0.1 & 0.19 & 0.03 \\
\hline $\mathrm{NaCl}$ & 4.33 & 0.29 & 0.78 & 0.38 & 0.11 & 0.23 & 0.04 \\
\hline $\mathrm{KCl}$ & 6.37 & 0.18 & 0.75 & 0.25 & 0.05 & 0.13 & 0.01 \\
\hline
\end{tabular}

направления [100] с изменениями параметра анизотропии и кривизны изоэнергетических поверхностей. Из табл. 3 видно, что в окрестности направления [100] при $\varphi=0$ и $\pi / 4$ для всех кристаллов коэффициенты $A^{t 2}(0, \varphi)$ имеют различные значения, т.е. претерпевают конечные разрывы. Как показал проведенный выше анализ, эти разрывы обусловлены различной кривизной изоэнергетических поверхностей для волновых векторов в плоскостях грани куба и диагональных плоскостях. Причем, в кристаллах первого типа при переходе от плоскости грани куба к диагональной плоскости меняются не только величины главных кривизн для медленных поперечных мод, но и их знак (тип кривизн) (см. табл. 3). Однако для кристаллов второго типа изоэнергетические поверхности для быстрых поперечных мод остаются выпуклыми, изменяется только величины кривизн при $\varphi=0$ и $\pi / 4$ (см. табл. 3). Максимальные величины разрывов коэффициенты $A^{t 2}(0, \varphi)$ достигают в кристаллах первого типа, входящих в четвертую область. Так, например, для Ge при $\theta \rightarrow 0$ коэффициенты $A^{t 2}(0,0) \cong 38$ и $A^{t 2}(0, \pi / 4) \cong 0.4$ отличаются на два порядка величины. Следует отметить, что для кристаллов первого типа нет корреляции между значением параметра анизотропии $k-1$ и величиной разрыва в коэффициентах усиления. Однако такая корреляция ясно прослеживается для кристаллов второго типа: чем выше обсолютное значение параметра анизотропии $|k-1|$, тем больше относительная величина разрывов в коэффициентах усиления в наравлениях типа [100] (см. табл. 3). Поскольку для них величины коэффициентов усиления меньше единицы, то абсолютные величины разрывов $A^{t 1}(0, \varphi)$ малы.

\section{4. Заключение}

Основные результаты работы могут быть сформулированы следующим образом:

1. Рассмотрены особенности распространения фононных импульсов в кубических кристаллах с различным типом анизотропии упругой энергии. Получено замкнутое аналитическое выражение для коэффициента усиления потока фононов. Проанализированы особенности его зависимостей от типов кривизны изоэнергетических поверхностей для всех акустических мод, а также от величины и знака параметров анизотропии.

2. Показано, что для волновых векторов в плоскости грани куба в коэффициентах усиления быстрой поперечной моды в полупроводниковых кристаллах первого типа преобладают эффекты фокусировки, и их значения больше единицы. Однако для металлических кристаллов первого типа $\mathrm{Na}, \mathrm{K}, \mathrm{Li}$ и $\mathrm{Cu}$ с максимальным значением параметра анизотропии $(k-1>1)$, коэффициенты усиления быстрой поперечной моды оказываются меньше единицы. Во всех кристаллах второго типа $(k-1<0)$ для медленной поперечной моды преобладают эффекты дефокусировки, и коэффициенты усиления оказываются меньше единицы.

3. Исследована связь величин разрывов коэффициентов усиления поперечных мод в окрестности направ- 
ления [100] с изменениями параметра анизотропии и кривизны изоэнергетических поверхностей. Показано, что в кристаллах первого типа при переходе от плоскости грани куба к диагональной плоскости меняются не только величины главных кривизн для медленных поперечных мод, но и их знак (тип кривизн). Однако для кристаллов второго типа изоэнергетические поверхности для быстрых поперечных мод остаются выпуклыми изменяется только величины кривизн.

Подводя итог анализу влияния фокусировки на распространение фононных мод в упруго анизотропных кристаллах, можно утверждать, что подход, основанный на коэффициенте усиления [5-12], не позволяет пока сделать количественные оценки для плотностей состояний квазипоперечных мод. Однако он находит широкое применение при анализе баллистического транспорта фононов и построения фононных изображений [5-12]. С другой стороны в работах $[18,20]$ нами предложен простой и наглядный способ оценки средних плотностей состояний (ПФС) для областей фокусировки и дефокусировки фононов, в котором изотропная среда также использовалась в качестве системы сравнения. Он дает достаточно грубую оценку влияния фокусировки на ПФС, но он свободен от отмеченных выше недостатков коэффициента усиления. Весомым аргументом в пользу развитого в работах $[18,20]$ метода оценки средних ПФС является хорошее согласие угловых зависимостей ПФС и длин свободного пробега: сектора максимумов обоих величин соответствуют областям фокусировки, а минимумов - областям дефокусировки фононов. Поэтому в дальнейшем при анализе влияния фокусировки поперечных фононов на фононный транспорт мы будем использовать этот метод.

\section{Список литературы}

[1] B. Taylor, H.J. Maris, C. Elbaum. Phys. Rev. Letter 23, 416 (1969).

[2] H.J. Maris. J. Acoust. Soc. Am. 50, 812 (1971).

[3] J.P. Wolfe. Imaging Phonons Acoustic Wave Propagation in Solids, Cambridge University Press, New York (1998), 411 p.

[4] B. Taylor. Phys. Rev. B, 3, 1462 (1971).

[5] Cz. Jasiukiewicz, T. Paszkiewicz, D. Lehmann. Z. Phys. B, 96, 213 (1994).

[6] E. Held, W. Klein, R.P. Huebener. Zeitschrift fur Physik B: Condensed Matter 75, 17 (1989).

[7] M. Lax, V. Narayanamurti. Phys. Rev. B, 22, 10, 4876 (1980).

[8] G.A. Northrop, J.P. Wolfe. Phys. Rev. B, 22, 12, 6196 (1980).

[9] J.A. Shields, J.P. Wolfe, S.I. Tamura. Z. Phys. B, 76, 295 (1989).

[10] J. Philip, K.S. Viswanathan. Phys. Rev. B, 17, 12, 4969 (1978).

[11] A.G. Every. Phys. Rev. B, 24, 6, 3456 (1981).

[12] C. Jasiukiewicz, T. Paszkiewicz. Acta physica polonica series A, 84, 459 (1993).

[13] A.G. Every, A.A. Maznev. Physics Letters A378, 3372 (2014).

[14] R. Anufriev, A. Ramiere, J. Maire, M. Nomura. Nature Communications 15505 (2017).
[15] L. Wang. The Journal of the Acoustical Society of America 123, 4140 (2008).

[16] I.A. Veres, D.M. Profunser, A.A. Maznev, A.G. Every. New Journal of Physics 14, 123015 (2012).

[17] И.Г. Кулеев, И.И. Кулеев. ФТТ 49, 3, 422 (2007).

[18] I.I. Kuleyev, S.M. Bakharev, I.G. Kuleyev, V.V. Ustinov. Phys. Status. Solidi. C, 14, 3-4, 1600263 (2017).

[19] I.G. Kuleyev, I.I. Kuleyev, I.Yu. Arapova. J. Phys.: Condens. Matter 22, 9, 095403 (2010).

[20] И.И. Кулеев, С.М. Бахарев, И.Г. Кулеев, В.В. Устинов. ФММ 118, 1, 12 (2017).

[21] Ф.И. Федоров. Теория упругих волн в кристаллах. М.: Наука (1965), 386 с.

[22] А.В. Погорелов. Дифференциальная геометрия (6-е издание). М.: Наука (1974), 176 с.

Редактор Т.Н. Василевская 